\title{
Nausea and vomiting in children and adolescents receiving intrathecal methotrexate: A prospective, observational study
}

\author{
Jacqueline Flank $^{1,2} \quad$ Komail Nadeem ${ }^{1,2} \quad$ Sheliza Moledina ${ }^{1}$ | Mila Khanna ${ }^{3}$ \\ Christina Schindera $^{4,5}$ | Angela Punnett ${ }^{6,7}$ | L. Lee Dupuis ${ }^{1,2,3}$
}

${ }^{1}$ Department of Pharmacy, The Hospital for Sick Children, Toronto, Canada

${ }^{2}$ Leslie Dan Faculty of Pharmacy, University of Toronto, Toronto, Canada

${ }^{3}$ Research Institute, The Hospital for Sick Children, Toronto, Canada

${ }^{4}$ Department of Pediatrics, University Children's Hospital, Basel, Switzerland

${ }^{5}$ Institute of Social and Preventive Medicine, University of Bern, Bern, Switzerland

${ }^{6}$ Division of Haematology/Oncology, Department of Paediatrics, The Hospital for Sick Children, Toronto, Canada

${ }^{7}$ Faculty of Medicine, University of Toronto, Toronto, Canada

Correspondence

L. Lee Dupuis, Research Institute, The Hospital for Sick Children, 555 University Ave., Toronto, ON M5G 1X8, Canada.

Email: lee.dupuis@sickkids.ca

\begin{abstract}
Background: The prevalence of nausea and vomiting after receipt of intrathecal methotrexate (ITMTX) in pediatric oncology patients is unknown.

Methods: Patients (4-18 years) about to receive IT-MTX were eligible to participate in this prospective, observational study. Patients received antiemetics as prescribed by their clinical team. Nausea severity (patient-assessed), timing of emetic episodes, and administration of antiemetics were recorded beginning immediately prior to IT-MTX administration, for the next $24 \mathrm{hr}$ (acute phase), and for a maximum of 7 additional days (delayed phase). Complete chemotherapy-induced nausea and vomiting (CINV) control was defined as no emetic episodes and no nausea.
\end{abstract}

Results: One hundred patients consented to participate in this study; 70 provided evaluable data (mean age: 8.3 years; range: 4.1-17.6). Most (94\%) received propofol-containing anesthesia for ITMTX administration. Most (89\%) received a 5-HT3 antagonist prior to IT-MTX. During the acute phase, 36 children (51\%) experienced complete CINV control, 67 (96\%) complete vomiting control, and 36 (51\%) complete nausea control. Severe acute phase nausea was reported by 12 children (17\%). During the delayed phase, 35 patients (50\%) experienced complete CINV control, 60 (86\%) complete vomiting control, and 36 (51\%) complete nausea control. Severe nausea was reported in the delayed phase by 27 (39\%) patients.

Conclusions: Most pediatric patients who received IT-MTX and prophylaxis with ondansetron or granisetron experienced complete acute and delayed vomiting control. However, nausea control was poor and severe nausea was reported by many children. Effective interventions to control nausea are needed.

KEYWORDS

chemotherapy, injections: spinal, methotrexate, nausea, pediatrics, vomiting

\section{INTRODUCTION}

Chemotherapy emetogenicity is the primary factor considered when selecting chemotherapy-induced nausea and vomiting (CINV) prophylaxis for children. ${ }^{1}$ Yet, the CINV experience of children and adolescents receiving specific chemotherapy is seldom documented. To date, no publications have evaluated nausea and vomiting in pediatric patients exclusively receiving intrathecal methotrexate (IT-MTX), a required component of acute lymphoblastic leukemia (ALL) treat-

Abbreviations: ALL, acute lymphoblastic leukemia; CIN, chemotherapy-induced nausea; CINV, chemotherapy-induced nausea and vomiting; CIV, chemotherapy-induced vomiting; IT-MTX, intrathecal methotrexate; IV, intravenous; PeNAT, Pediatric Nausea Assessment Tool; PO, oral; TIT, triple intrathecal therapy ment. Without this information, it is difficult to apply the recommendations of clinical practice guidelines for the prevention of CINV 2,3 and to optimize CINV control. In this study, we used a validated, pediatric nausea self-assessment measure the (PeNAT) ${ }^{4}$ to describe the prevalence of acute and delayed CINV in pediatric patients with ALL receiving IT-MTX.

\section{2 | METHODS}

This prospective, single-center, observational study was approved by the Research Ethics Board at SickKids. Each patient or their guardian provided informed consent or assent to study participation. 


\section{1 | Patients}

Patients 4-18 years of age who were about to receive IT-MTX within the second or later maintenance cycle of an ALL treatment protocol, were English-speaking with an English-speaking parent, and had no physical or cognitive impairment that precluded the use of the PeNAT ${ }^{4}$ were eligible to participate. Patients planned to receive chemotherapy other than oral (PO) 6-mercaptopurine, $\mathrm{PO}$ prednisone, or dexamethasone or intravenous (IV) vincristine during the week following the ITMTX dose were excluded. Patients received antiemetics as ordered by the clinical team. Patients participated only once.

\subsection{Data collection}

Patient's demographic data, chemotherapy and antiemetic agents given in hospital or clinic during the study period, and medication given during the lumbar puncture procedure were abstracted from the health record. Patients or their parent were asked if they had a history of uncontrolled chemotherapy-induced vomiting (CIV) defined as a previous hospital admission or the prolongation of a hospital admission for management of vomiting following chemotherapy administration. Antiemetic medication given at home during the study period was recorded by the patient or parent on a diary provided.

\subsection{CINV assessment}

Patients and guardians recorded the patient's PeNAT scores and the time of each vomit and retch on the provided diary. Vomiting was defined as the expulsion of any stomach contents by the mouth. Retching was defined as an attempt to vomit that is not productive of any stomach contents.

A member of the study team administered the PeNAT to the patient once before IT-MTX administration and asked the patient and their parent to report the patient's experience with nausea, vomiting, and retching over the previous $24 \mathrm{hr}$. The parent and patient were then instructed on the administration of the PeNAT and on the completion of the diary. Diaries were to be completed on each day of the acute and delayed phases. The acute phase started with the administration of the IT-MTX dose within the study period and continued for $24 \mathrm{hr}$ or, if vincristine was administered, for $24 \mathrm{hr}$ after the administration of the vincristine dose. The delayed phase started when the acute phase ended and continued for 7 days ( $168 \mathrm{hr}$ ). During the acute and delayed phases, each patient was asked to rank the severity of his or her nausea at minimum twice daily (on rising in the morning and at bedtime) plus at any other time that they felt nauseated or their guardian believed them to feel nauseated. If the patient could not record their data on the diary or chose not to, guardians were instructed to record the nausea severity as ranked by their child without interpretation.

A member of the study team contacted each family up to three times during the study period to answer any questions and to remind them to complete and return the diary.

\subsection{Statistical analysis}

The primary endpoint of this descriptive study was complete control of CINV during the acute phase (Table 1). A sample size of 


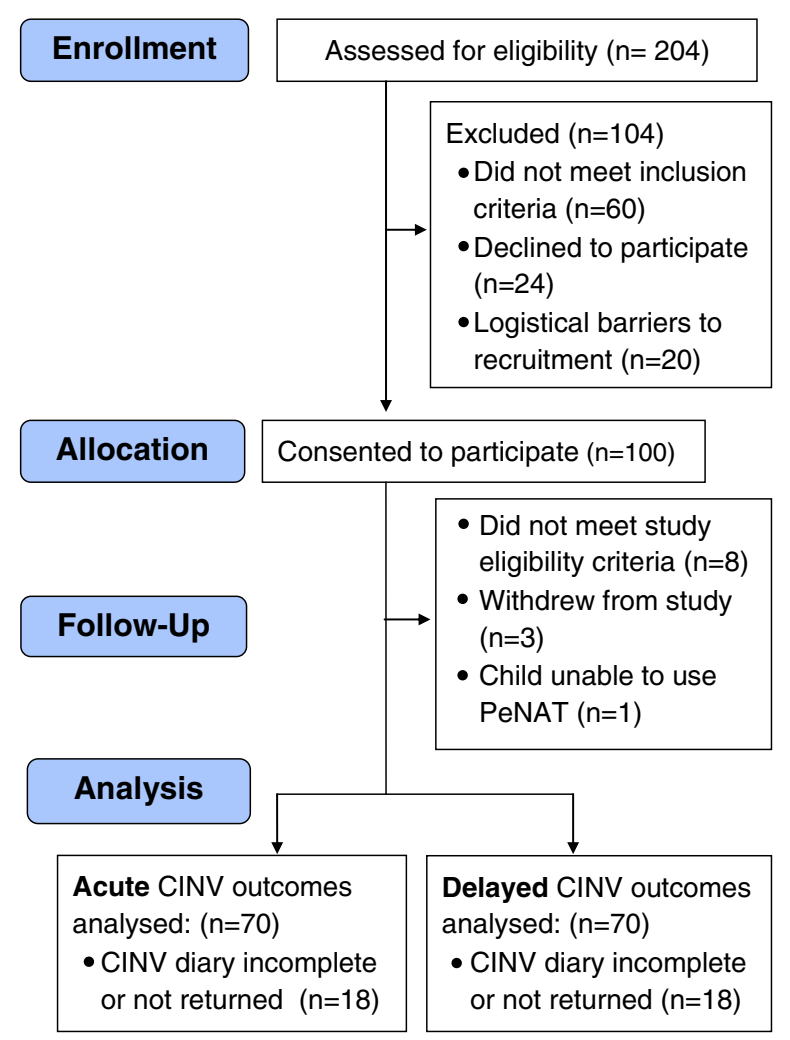

FIGURE 1 Study flow diagram

100 patients was chosen to permit the description of CINV prevalence with confidence and allowed for an unknown proportion of children who might not provide evaluable data. The secondary study endpoints were complete CIV and chemotherapy-induced nausea (CIN) control during the acute phase and complete CINV, CIV, and CIN control during the delayed and overall phases. The overall phase is comprised of both the acute and delayed phases. The proportions of patients who experienced complete, partial, and uncontrolled acute CINV control in the acute, delayed, and overall phases were described.

Differences between groups were assessed using chi-square or nonparametric analyses as appropriate (SAS Enterprise Guide 6.100, SAS Institute Inc., Cary, NC, USA).

\section{RESULTS}

\section{1 | Patients}

Two hundred four patients were screened from May 7, 2012, through October 2, 2015, and 124 were invited to participate in the study. Of the 88 patients who initiated the study, 70 returned completed diaries. Patients who did not return completed diaries did not differ from patients who did return completed diaries in terms of mean age $(7.6 \pm 4.2$ vs. $8.3 \pm 3.3$ years; $P=0.14$ ) or sex (female/male: $5 / 13$ vs $19 / 51 ; P=0.23)$. The study flow diagram is presented in Figure 1.

Patient and treatment characteristics are presented in Table 2. Most patients received ondansetron or granisetron as CINV prophylaxis prior to IT-MTX (62/70), propofol as anesthetic (67/70), and vincristine (69/70) on the same day of IT-MTX. Approximately one-third of patients received antiemetic agents (ondansetron/granisetron: 18; dimenhydrinate: 2; nabilone: 1 ) later in the acute phase. Almost all patients received PO 6-mercaptopurine (69/70) and PO MTX (68/70) during the delayed phase.

\section{2 | CINV Control}

CINV, CIV, and CIN control rates reported in the acute, delayed, and overall phases are presented in Table 3. During the acute phase, 36 patients (51\%) experienced complete CINV control, 67 (96\%) complete vomiting control, and 36 (51\%) complete nausea control. Severe acute nausea was reported by 12 children (17\%). In the delayed phase, 35 children (50\%) experienced complete CINV control, 60 (86\%) complete vomiting control, and 36 (51\%) complete nausea control. Severe nausea was reported in the delayed phase by 27 (39\%) children.

\section{4 | DISCUSSION}

In this prospective study, we found that pediatric patients who received ondansetron or granisetron prior to IT-MTX experienced excellent vomiting control in the acute phase. Acute phase nausea, however, was less well controlled and poor acute CINV control was primarily driven by nausea. This pattern persisted in the delayed and overall phases.

Nausea and vomiting following intrathecal chemotherapy administration may stem from the intrathecal chemotherapy itself, the act of lumbar puncture and concurrent medication including anesthetic agents, analgesics, and IV/PO chemotherapy. Five studies have described vomiting in pediatric patients following intrathecal chemotherapy administration. ${ }^{5-9}$ One randomized patients to receive midazolam plus one of four possible combinations given once prior to administration of unspecified intrathecal chemotherapy: fentanyl or placebo plus ondansetron or placebo. ${ }^{9}$ All children received propofol. Patients who received ondansetron reported fewer episodes of vomiting or retching during the $12 \mathrm{hr}$ after lumbar puncture (overall reduction of 4.664; $P<0.0001$ ). Three studies evaluated vomiting after administration of triple intrathecal therapy (TIT; cytarabine, MTX, and hydrocortisone) to pediatric patients. ${ }^{6,7,10}$ In the first, vomiting was evaluated in a subset of 16 patients after 27 doses of TIT where no antiemetic prophylaxis had been given. ${ }^{10}$ The mean number of vomiting episodes reported in the 7 days after TIT administration in this subset was $8.3 \pm 12.2$. The same authors later described vomiting control in 63 patients aged $1-17$ years who received TIT. ${ }^{6}$ Patients received either no antiemetic prophylaxis or ondansetron IV/PO. No general anesthetic agents or opioids were administered. Most (78\%) of the patients who received no antiemetic prophylaxis vomited during the week following TIT administration, whereas this rate was 31 and $44 \%$ in patients who received IV and $\mathrm{PO}$ ondansetron, respectively. Vomiting was described as typically beginning 3-4 hr after TIT administration and rarely lasting longer than $24 \mathrm{hr}$. Lastly, Parker et al. have described vomiting control following 146 intrathecal chemotherapy doses in 26 pediatric patients randomized to receive placebo, low-dose IV ondansetron, or high-dose IV ondansetron. ${ }^{7}$ Most (22/26) patients 
TABLE 2 Characteristics of the 70 evaluable patients and their treatment

\begin{tabular}{|c|c|}
\hline \multicolumn{2}{|c|}{ Patient Characteristics } \\
\hline Mean age, years (range) & $8.3(4.1-17.6)$ \\
\hline Sex, no. of patients (male:female) & $51: 19$ \\
\hline Anticipatory nausea or vomiting prior to IT-MTX, no. of patients (\%) & $12(17)$ \\
\hline \multicolumn{2}{|c|}{ Treatment Characteristics } \\
\hline \multicolumn{2}{|l|}{ Receipt of concurrent corticosteroid, no. of patients } \\
\hline Prednisone & 32 \\
\hline Dexamethasone & 30 \\
\hline None & 8 \\
\hline \multicolumn{2}{|l|}{ Anesthetic administered, no. of patients } \\
\hline \multicolumn{2}{|l|}{ Antiemetic administered before IT-MTX, no. of patients } \\
\hline Ondansetron/granisetron & 62 \\
\hline None $^{a}$ & 8 \\
\hline \multicolumn{2}{|l|}{ Antiemetic administered during the delayed phase, no. of patients } \\
\hline Ondansetron/granisetron & 11 \\
\hline Dimenhydrinate & 3 \\
\hline Dexamethasone & 1 \\
\hline None & 55 \\
\hline
\end{tabular}

IT-MTX, intrathecal methotrexate; CIV, chemotherapy-induced vomiting.

${ }^{a}$ Of the eight children who did not receive an antiemetic, seven were given ondansetron or granisetron immediately after the procedure.

TABLE 3 Nausea and vomiting control reported by 70 children receiving IT-MTX

\begin{tabular}{|c|c|c|c|}
\hline Level of control & CIV & CIN & CINV \\
\hline \multicolumn{4}{|c|}{ Acute phase, no. of patients (\%) } \\
\hline Complete control & $67(96)$ & $36(51)$ & $36(51)$ \\
\hline Partial control & $3(4)$ & $22(31)$ & $22(31)$ \\
\hline Uncontrolled & $0(0)$ & $12(17)$ & 12 (17) \\
\hline \multicolumn{4}{|c|}{ Delayed phase, no. of patients (\%) } \\
\hline Complete control & $60(86)$ & $36(51)$ & $35(50)$ \\
\hline Partial control & $9(13)$ & $7(10)$ & $8(11)$ \\
\hline Uncontrolled & $1(1)$ & $27(39)$ & 27 (39) \\
\hline \multicolumn{4}{|c|}{ Overall phase, no. of patients (\%) } \\
\hline Complete control & $40(57)$ & $26(37)$ & $26(37)$ \\
\hline Partial control & $15(21)$ & $13(19)$ & $13(19)$ \\
\hline Uncontrolled & $15(21)$ & $31(44)$ & $31(44)$ \\
\hline
\end{tabular}

received TIT and most doses were accompanied by midazolam plus ketamine. The proportions of patients who did not vomit during the $48 \mathrm{hr}$ after intrathecal chemotherapy and who received placebo, lowdose ondansetron, or high-dose ondansetron were 37,73 , and $86 \%$, respectively.

Traivaree et al.conducted a randomized, cross-over, double-blind trial to evaluate a single dexamethasone dose to prevent vomiting associated with intrathecal chemotherapy in 33 children (aged 2.214.2 years) given ketamine sedation. ${ }^{8}$ All but one patient received IT-MTX. In the $24 \mathrm{hr}$ after intrathecal chemotherapy administration, vomiting rates in the dexamethasone group were significantly lower compared with those in the placebo group ( $15 \mathrm{vs.} 49 \%$; $P=0.02$ ).

As stated above, coadministration of anesthetic and other agents during intrathecal chemotherapy administration may increase the risk of emesis of intrathecal chemotherapy. Of the pediatric risk factors identified for postoperative vomiting, two are relevant in the context of intrathecal chemotherapy administration: age greater than 3 years and history of postoperative vomiting in the patient, parent, or sibling. ${ }^{11}$ Since all patients in our population were older than 3 years and we did not have information relating to familial history, we could not explore these relationships.

The rate of complete acute CIV control observed in our study was higher than has been reported in children receiving dexamethasone or ondansetron prior to intrathecal chemotherapy administration. Differences may be due, at least in part, to the anesthetic agent (e.g., propofol vs. ketamine). For example, propofol has a very low emetic risk and is, therefore, the preferred anesthetic for patients at high risk of postoperative vomiting. ${ }^{11}$ In addition, unlike other studies where patients received a single ondansetron dose, several patients in our study received more than one ondansetron dose during the acute phase. The corticosteroid administered as part of the leukemia treatment may also have conveyed an antiemetic effect. It is also possible 
that specific chemotherapy agents may be more or less emetogenic than MTX.

Interpretation of our study findings regarding CINV in the delayed phase following IT-MTX administration is limited by the administration of $\mathrm{PO}$ chemotherapy during this period. Although the rates of vomiting and nausea reflect the experience of our patients, symptoms are likely attributable to both IT-MTX and PO chemotherapy. Furthermore, participation in our study may have been subject to selection bias; it is possible that patients may have been more or less apt to participate depending on their prior experience with CINV.

In conclusion, acute phase CIV control in children receiving IT-MTX with propofol anesthesia and ondansetron or granisetron is excellent. Our study, the first to use a validated, pediatric, self-report measure to evaluate nausea severity in children receiving intrathecal chemotherapy, found that severe nausea is a common experience of children who receive IT-MTX. Effective antinausea interventions must be identified to optimize CINV control in children.

\section{ACKNOWLEDGMENTS}

Partial funding from the CIHR Summer Student Research Program is acknowledged. The generosity of the parents and patients who participated in this study is acknowledged with thanks. The assistance of Mary Arsanious with study procedures is also acknowledged.

\section{CONFLICT OF INTEREST}

The authors declare that there is no conflict of interest.

\section{REFERENCES}

1. Dupuis L, Boodhan S, Sung L, et al. Guideline for the classification of the acute emetogenic potential of antineoplastic medication in pediatric cancer patients. Pediatr Blood Cancer. 2011;57:191-198.

2. Dupuis LL, Boodhan S, Holdsworth M, et al. Guideline for the prevention of acute nausea and vomiting due to antineoplastic medication in pediatric cancer patients. Pediatr Blood Cancer. 2013;60:1073-1082.

3. Patel P, Robinson P, Thackray J, et al. Guideline for the prevention of acute chemotherapy-induced nausea and vomiting in pediatric cancer patients: a focused update. Pediatr Blood Cancer. 2017;00:e26542. https://doi.org/10.1002/pbc.26542

4. Dupuis LL, Taddio A, Kerr EN, Kelly A, MacKeigan L. Development and validation of a pediatric nausea assessment tool (PeNAT) for use by children receiving antineoplastic agents. Pharmacotherapy. 2006;26:1221-1231.

5. Holdsworth MT, Raisch DW, Frost J. Acute and delayed nausea and emesis control in pediatric oncology patients. Cancer. 2006;106:931940.

6. Holdsworth MT, Raisch DW, Winter SS, Chavez CM. Assessment of the emetogenic potential of intrathecal chemotherapy and response to prophylactic treatment with ondansetron. Support Care Cancer. 1998;6(2):132-138.

7. Parker RI, Prakash D, Mahan RA, Giugliano DM, Atlas MP. Randomized, double-blind, crossover, placebo-controlled trial of intravenous ondansetron for the prevention of intrathecal chemotherapyinduced vomiting in children. J Pediatr Hematol/Oncol. 2001;23(9):578581.

8. Traivaree C, Torcharus K, Lumkul R, Kormoltri C, Charuluxananan S. Efficacy of intravenous dexamethasone for the prevention of vomiting associated with intrathecal chemotherapy and ketamine sedation in children: a randomized, double-blinded, crossover, placebo-controlled trial. Asian Biomed. 2011;5(4):441-448.

9. Nagel K, Willan A, Lappan J, Korz L, Buckley N, Barr R. Pediatric oncology sedation trial (POST): a double-blind randomized study. Pediatr Blood Cancer. 2008;51:634-638.

10. Holdsworth MT, Raisch DW, Duncan MH, Chavez CM, Leasure MM. Assessment of chemotherapy-induced emesis and evaluation of a reduced-dose intravenous ondansetron regimen in pediatric outpatients with leukemia. Ann Pharmacother. 1995;29:16-21.

11. Gan TJ, Diemunsch P, Habib AS, et al. Consensus guidelines for the management of postoperative nausea and vomiting. Anesth Analg. 2014;118(1):85-113. 